\title{
LAMP-5 is an essential inflammatory-signaling regulator and novel immunotherapy target for mixed lineage leukemia-rearranged acute leukemia

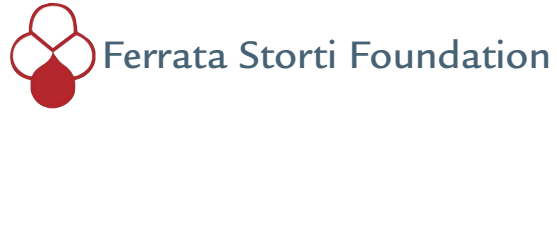

\author{
Gabriel Gracia-Maldonado,,2 Jason Clark,, ${ }^{1,2}$ Matthew Burwinkel,,,2 \\ Brenay Greenslade, ${ }^{1,3}$ Mark Wunderlich, ${ }^{1,4}$ Nathan Salomonis, ${ }^{5,7}$ Dario Leone, ${ }^{6}$ \\ Evelina Gatti, ${ }^{6}$ Philippe Pierre, ${ }^{6}$ Ashish R. Kumar ${ }^{1,2,7}$ and Lynn H. Lee $e^{1,3,7}$
}

${ }^{1}$ Cancer and Blood Diseases Institute, Cincinnati Children's Hospital Medical Center, Cincinnati, $\mathrm{OH}, \mathrm{USA} ;{ }^{2}$ Division of Bone Marrow Transplantation and Immune Deficiency, Cincinnati, Children's Hospital Medical Center, Cincinnati, OH, USA; ${ }^{3}$ Division of Oncology, Cincinnati Children's Hospital Medical Center, Cincinnati, OH, USA; ${ }^{4}$ Division of Experimental Hematology and Cancer Biology, Cincinnati, Children's Hospital Medical Center, Cincinnati, OH, USA; 'Division of Biomedical Informatics, Cincinnati Children's Hospital Medical Center, Cincinnati, OH, USA; ${ }^{6}$ Aix Marseille Université, CNRS, INSERM, Centre d'Immunologie de Marseille-Luminy (CIML), Marseille, France and ${ }^{7}$ Department of Pediatrics, University of Cincinnati School of Medicine, Cincinnati, OH, USA

\section{ABSTRACT}

A lthough great advances have been made in understanding the pathobiology of mixed lineage leukemia-rearranged (MLL-r) leukemias, therapies for this leukemia have remained limited, and clinical outcomes remain bleak. In order to identify novel targets for immunotherapy treatments, we compiled a lineage-independent MLL-r leukemia gene signature using publicly available data sets. Data from large leukemia repositories were filtered through the in silico human surfaceome, providing a list of highly predicted cell surface proteins overexpressed in MLL-r leukemias. LAMP5, a lysosomal associated membrane protein, is expressed highly and specifically in MLL-r leukemia. We found that $L A M P 5$ is a direct target of the oncogenic MLL-fusion protein. LAMPS depletion significantly inhibited leukemia cell growth in vitro and in vivo. Functional studies showed that LAMP-5 is a novel modulator of innate-immune pathways in MLL-r leukemias. Downregulation of LAMP5 led to inhibition of NF- $\mathrm{KB}$ signaling and increased activation of type-1 interferon signaling downstream of Toll-like receptor/interleukin 1 receptor activation. These effects were attributable to the critical role of LAMP-5 in transferring the signal flux from interferon signaling endosomes to pro-inflammatory signaling endosomes. Depletion of IRF7 was able to partially rescue the cell growth inhibition upon $L A M P 5$ downregulation. Lastly, LAMP-5 was readily detected on the surface of MLL-r leukemia cells. Targeting surface LAMP-5 using an antibody-drug conjugate leads to significant cell viability decrease specifically in MLL-r leukemias. Overall, based on the limited expression throughout human tissues, we postulate that LAMP-5 could potentially serve as an immunotherapeutic target with a wide therapeutic window to treat MLL-r leukemias.

\section{Introduction}

Translocations in the mixed lineage leukemia (MLL) gene account for $10 \%$ of all human leukemias and are associated with pediatric, adult, and therapy-related cases. In infants, around $80 \%$ of acute lymphoid leukemia (ALL) and $35 \%-50 \%$ of acute myeloid leukemia (AML) cases carry a translocation in the MLL gene. ${ }^{1}$ However, despite improvements in conventional chemotherapy treatments for leukemia, patients with MLL-rearranged leukemia (MLL-r) have a poor response to treatment (ogica 2022

Volume 107(3):803-815

Correspondence:

LYNN H. LEE

lynn.lee@cchmc.org

ASHISH R. KUMAR

ashish.kumar@cchmc.org

Received: April 30, 2020.

Accepted: April 2, 2021.

Pre-published: April 29, 2021.

https://doi.org/10.3324/haematol.2020.257451

(C)2022 Ferrata Storti Foundation

Material published in Haematologica is covered by copyright. All rights are reserved to the Ferrata Storti Foundation. Use of published material is allowed under the following terms and conditions:

https://creativecommons.org/licenses/by-nc/4.0/legalcode. Copies of published material are allowed for personal or internal use. Sharing published material for non-commercial purposes is subject to the following conditions:

https://creativecommons.org/licenses/by-nc/4.0/legalcode, sect. 3. Reproducing and sharing published material for commercial purposes is not allowed without permission in writing from the publisher. 
and poor prognosis., ${ }^{2,3}$ Immunotherapy strategies have proven effective in multiple blood cancers, mainly targeting lineage-specific proteins like CD19 (blinatumomab, tisagenlecleucel) and CD33 (gemtuzumab), abundantly expressed in ALL and AML patients, respectively. ${ }^{4}$ However, mounting evidence in recent clinical trials and case reports have shown that patients with MLL-rearrangements frequently relapse after treatment with CD19 immunotherapies, arising as AML or mixed phenotype acute leukemia (MPAL). ${ }^{5-12}$ The exact mechanism of lineage switch induced by CD19 immunotherapies is still unclear.

One approach to overcome the lineage switching is to develop MLL-r specific immunotherapies targeting cell surface proteins essential for the survival of MLL-r leukemias. Recently, NG2/CSPG4 and CD133/PROM1 have been shown to be promising MLL-r specific immunotherapy targets, however, these targets are restricted to lymphoid lineage, increasing the potential for lineage switching within the leukemic population. ${ }^{13-15}$ Gene-expression profiling based on underlying cytogenetic mutations is one way to identify proteins that are overexpressed and thus might be essential for the propagation of the specific leukemia. ${ }^{16,17}$ Both AML and ALL with MLL-rearrangements share a common gene signature that is distinct from that of MLL-germline (MLL-G) leukemias. ${ }^{18}$ Most of the well-studied and validated MLL$r$ gene targets however are DNA binding proteins like the HOXA gene cluster and its co-factor MEIS1, ${ }^{19,20}$ which are not suitable targets for immunotherapy.

In several of the published gene-expression studies, we found $L A M P 5$ significantly and specifically overexpressed in MLL-r leukemias. ${ }^{18,21-23}$ LAMP-5 is a member of the lysosome-associated membrane protein (LAMP) family. In contrast to other LAMP proteins which show widespread expression, Lamp5 expression in mice is confined to several regions of the postnatal brain. In neurons, the protein was found to recycle between the plasma membrane and a non-classical endosomal vesicle. ${ }^{24-26}$ In humans, aside from its conserved expression in the brain, $L A M P 5$ is specifically expressed in plasmacytoid dendritic cells ( $\mathrm{pDC}$ ). ${ }^{27}$ Upon activation of $\mathrm{pDC}$, LAMP-5 aids in the transport of Toll-like receptor 9 (TLR9) from early endosomal to lysosomal signaling vesicles, thereby regulating type 1 interferon (IFN-1) and pro-inflammatory signaling respectively, downstream of TLR9 activation. ${ }^{28}$ Importantly, results of in silico modeling predict LAMP-5 as a cell surface protein. ${ }^{29}$ In this report, we demonstrate $L A M P 5$ as being highly expressed and essential for MLL$r$ leukemias through the regulation of innate immune signaling and describe its potential as a target for MLL-r specific immunotherapy.

\section{Methods}

\section{Cell lines and primary patient-derived xenograft cells}

Human leukemia cell lines were maintained in Iscove's Modified Dulbecco Medium (IMDM) or Roswell Park Memorial Institute (RPMI) 1640 medium supplemented with 10\% fetal bovine serum (FBS), 1\% penicillin, and 1\% streptomycin.

Fully de-identified primary cells were obtained from the Cincinnati Children's Hospital Medical Center Biorepository. Cells were cultured in IMDM supplemented with 20\% FBS and $10 \mathrm{ng} / \mathrm{mL}$ human cytokines including SCF, FLT3-ligand, thrombopoietin, IL-3, and IL-6.

\section{Animal experiments}

All animal experiments were carried out in accordance with the guidelines of the Institutional Animal Care and Use Committee (IACUC). For xenograft experiments with MV4;11 and MLL-AF10 primary patient cells, immunocompromised NOD-Rag ${ }^{\text {null }} I L 2 \gamma^{\text {null }}$ (NRG) (Jackson Laboratories, stock no. 007799) recipient mice were conditioned with busulfan and transplanted 24 hours later. In xenograft experiments bone marrow samples were collected 4 weeks after transplantation as well as when signs of leukemia were present; bone marrow aspirates were analyzed via flow cytometry for the presence of human $\mathrm{CD} 45^{+}$cells and the presence of short hairpin RNA (shRNA)-transduced Venus+ cells

\section{Retroviral and lentiviral transductions}

Retroviral and lentiviral supernatants were generated by transfection of HEK293T cells using the FuGENE 6 reagent (Promega) or Lipofectamine 3000 (Thermo Fisher Scientific) according to the manufacturer's recommendations.

All lentiviral shRNA constructs were purchased from Millipore Sigma. Cells transduced with constructs containing a fluorescent marker (Venus) were sorted 4-5 days after transduction by using MoFlo XDP (Beckman Coulter), FACSAria (BD Biosciences), or a SONY SH800S (Sony Biotechnology).

\section{Flow cytometry}

For apoptosis assays, cells were incubated with allophycocyanin (APC)-conjugated annexin V (BD Biosciences) for 15 minutes at room temperature in $1 \mathrm{X}$ annexin $\mathrm{V}$ binding buffer $(\mathrm{BD}$ Biosciences) followed by staining with 7-aminoactinomycin D (7$\mathrm{AAD})$ (eBioscience). For surface LAMP-5 detection, cells were incubated with $3 \mu \mathrm{g}$ anti-human LAMP-5 therapeutic antibody overnight and then stained with anti-mouse IgG1-PE. Data were acquired on a BD FACSCanto analyzer and results were analyzed using FlowJo Version 10 (BD Biosciences).

\section{Colony-forming unit assays}

Transduced human cells were sorted 4-5 days after transduction and were cultured in methylcellulose-containing media (StemCell Technologies, H4434). Colonies were scored 10-14 days after plating.

\section{Real-time- and quantitative real-time polymerase chain reaction}

Total RNA was extracted from human puromycin-selected or sorted $\mathrm{Venus}^{+}$cells using the RNeasy Mini kit (OIAGEN). For quantitative real-time polymerase chain reaction (RT-PCR) 5-10 ng of cDNA was analyzed using iTaq Universal SYBR Green Supermix (Bio-Rad) or PowerUP SYBR Green (Thermo Fisher Scientific) in a StepOnePlus RT-PCR machine (Applied Biosystems).

\section{Cell viability}

MOLM-13, RS4;11, THP-1, and Kasumi-1 cells were plated at 10,000 cells per well in a 96 -well plate. Cells were incubated with LAMP-5 therapeutic antibody (Creative Biolabs) and anti-mouse immunoglobulin G (IgG) Fc-DM1 antibody with non-cleavable linker (Moradec) at $5 \mathrm{ng} / \mathrm{uL}$ and $1 \mathrm{ng} / \mathrm{uL}$ final concentrations, respectively. In order to measure cell viability CellTiter-Gloß 2.0 Cell Viability Assay (Promega) was used following the manufacturer's protocol.

\section{Immunofluorescence}

Cells seeded on alcian blue-treated coverslips were fixed with $3.5 \%$ paraformaldehyde and permeabilized with $0.05 \%$ saponin. Cells were stained overnight with primary antibodies against 
LAMP-5, MYD88, and LAMP-1. Immunofluorescence and confocal microscopy were performed with a Zeiss LSM580 63x objective and accompanying imaging software.

\section{Statistics}

The statistical methodology used, and sample sizes are described in the individual Figure legends. $t$-tests were two-tailed unless otherwise stated. Results are presented as mean \pm standard error of the mean (SEM) unless otherwise stated. A two-sided time-stratified Cochran-Mantel-Haenszel was used for the Kaplan-Meier Survival analysis. ROC curves were used to determine the diagnostic utility of LAMP5 mRNA. The sensitivity and specificity were identified at the optimal cutoff point that was chosen at which Youden's index was maximal. A significance level cutoff of 0.05 was used unless otherwise stated. Statistical analysis was performed using GraphPad Prism.

More detailed information on the materials and methods used can be found in the Online Supplementary Appendix.

\section{Results}

\section{LAMP5 is highly expressed in mixed lineage leukemia-rearranged leukemias and is a direct target of the mixed lineage leukemia-fusion protein}

In order to determine genes that are highly expressed in AML and ALL with MLL-rearrangements, we compared recently published RNA sequencing (RNA-seq) studies that identified differentially expressed genes between MLL-r and MLL-G leukemias in both AML and B-ALL samples ${ }^{30,31}$ (Figure 1A). Twenty-seven genes were commonly overexpressed in MLL-r ALL and AML (Online Supplementary Table S1). Using the in silico human surfaceome tool (http://wlab.ethz.ch/surfaceome) five of these 27 genes were predicted to be expressed on the cell surface $^{29}$ (Online Supplementary Table S1). Of the five predicted proteins, LAMP-5 stood out for being present in multiple previous MLL-r leukemia gene expression studies $^{17,18,21-23}$ (Online Supplementary Figure S1A and B). We further validated the specificity of LAMP5 expression in MLL-r leukemias by analyzing the 1,109 pediatric leukemia patient samples from the St.Jude PeCan Portal which revealed LAMP5 as significantly overexpressed in $92 \%$ of ALL and $72 \%$ of AML with MLL-rearrangements $^{16}$ (Figure 1B). In order to determine if LAMP5 expression could discriminate between MLL-r leukemia and MLL-G leukemia patients, we performed a receiving operating curve (ROC) analysis. LAMP5 achieved a statistically significant area under the curve (AUc) score in both the microarray innovations in both the (MILE) (GSE13159) and the St. Jude PeCan datasets, with high sensitivity and specificity at the optimal cutoff points (Figure 1C). Further, a Kaplan-Meier survival analysis of B-ALL and AML patients correlated higher expression of LAMP5 with poor survival (Online Supplementary Figure $S 2 A$ and $B)$. At the protein level, patient-derived xenograft (PDX) pediatric AML and ALL samples show high expression of LAMP-5 only in the MLL-r samples as compared to MLL-G (Figure 1D). Similar results were seen in human MLL-r AML and ALL cells lines (MOLM13, MV4;11, THP-1, and RS4;11) at the mRNA (Figure 1E) and protein (Figure 1F) levels as compared to MLL-G leukemia cell lines (HL-60, Kasumi-1, K562, REH, and $\mathrm{RCH}-\mathrm{ACV}$ ) and normal human CD34-enriched cord blood cells (CB-CD34+ cells). Translocations of the MLL locus generate MLL fusion proteins (MLL-FP) which activate transcription of downstream target genes. ${ }^{32,33}$ In order to determine if LAMP5 expression was dependent on the MLL-FP, we transformed CB-CD34+ cells with a retrovirus carrying a tetracycline-repressible MLL-AF9 construct. Treatment of transformed cells with doxycycline led to a simultaneous reduction in the levels of both MLL-AF9 and LAMP5 (Figure 1G). in order to determine if the MLL-FP directly activates the LAMP5 gene locus, we interrogated previously published MLL-FP chromatin immunoprecipitation sequencing (ChIP-seq) datasets derived from the SEM, RS4;11, MV4;11, THP-1, and ML2 cell lines, CD34 ${ }^{+}$cells transformed with FLAG-MLL-Af4, and primary patient sample. ${ }^{32-36}$ Almost all cell lines exhibited peaks within the LAMP5 promoter region, suggesting direct binding of the MLL-FP (as evidenced by coincident signal in both MLL and fusion partner ChIP-seq tracks). Additionally, there was accompanying significant enrichment of H3K79me2 and H3K79me3 along the gene body, further supporting our hypothesis that LAMP5 undergoes transcriptional activation in MLL-r leukemia via direct targeting by the MLL-FP complex (Figure 1H). In mice, Lamp5 does not show any expression in blood, as it does in humans (Online Supplemental Figure S3A and B). Furthermore, we did not detect upregulation of Lamp5 in mouse models of MLL-AF9, E2A-HLF, and AML1-ETO leukemia, hence we focused our studies exclusively on human cells (Online Supplemental Figure S3C).

\section{LAMP-5 is required for in vitro and in vivo leukemia cell survival}

The ideal immunotherapy target should be essential for the survival of MLL-r leukemias. In order to test the functional role of LAMP-5 in MLL-r leukemia, we transduced both MLL-r leukemia (MOLM-13, MV4;11, RS4;11, THP-1) and MLL-G leukemia cells (Kasumi-1 and REH) with lentiviral shRNA vectors targeting LAMP5. We obtained efficient knockdown of $L A M P 5$ with two independent hairpins as compared to non-targeting control (NT) (Online Supplementary Figure S4A). Upon LAMP5 depletion, we observed a significant reduction of cell growth in MLL-r leukemia cell lines (Figure 2A), while no effect was seen in Kasumi-1 and REH (Figure 2B). Additionally, LAMP5 knockdown led to a significant decrease in colony-forming units (CFU) in the MLL-r leukemia cell lines (Figure 2C) suggesting an effect on the clonogenicity of these cells. Furthermore, LAMP5 knockdown led to apoptosis in MLL-r leukemia cells, as evident by a significant increase in annexin $\mathrm{V}$ and 7-AAD double-positive staining (Figure 2D; Online Supplementalry Figure S4B). We next sought to determine the role of LAMP-5 in leukemia propagation in vivo. MV4;11 cells were transduced with shLAMP5-2 or NT control followed by transplantation into immunocompromised NOD-Rag $1^{\text {null }} I L 2 r \gamma^{\text {ull }}$ (NRG) mice (Figure 2E). In the bone marrow, both groups showed similar human cell engraftment based on human CD45 expression. On the other hand, the transduced Venus ${ }^{+}$fraction was significantly reduced in shLAMP5-2 compared to shNT mice 4 weeks after transplantation (Figure 2F, left panels). We repeated this experiment using cells from an AML PDX with MLL-r (MLL-AF10) leukemia. We again observed a significant reduction in the proportion of Venus ${ }^{+}$cells with LAMPS knockdown compared to NT control (Figure 2F, right panels). Overall, these data underscore a critical role for LAMP5 in the growth of MLL-r leukemia cells. 
LAMP-5 is required for activation of Toll-like receptor/interleukin 1 receptor signaling in leukemia

Acute leukemias exhibiting constitutive activation of innate immune signaling pathways have been characterized as having a pro-inflammatory profile which is required for their survival. ${ }^{37}$ These physiologic cellular systems involve TLR/IL-1R signaling and culminate in the release of pro-inflammatory cytokines via NF- $\mathrm{KB}$ and/or of type I interferons (IFN-1). ${ }^{38}$ Recent studies reveal heightened activation of NF- $\kappa B$ signaling in MLL-r leukemia compared to other leukemias. ${ }^{39}$ Furthermore, MLL-r leukemias have been shown to require the TLR/IL$1 \mathrm{R}$ signaling pathway to survive, through degradation of the wild-type MLL protein, allowing the MLL-FP to bind to its target genes without restriction. ${ }^{40}$ Recently, Combes et al. showed that LAMP-5 plays an important role in controlling the subcellular location of TLR9 after activation in human pDC. Upon activation of TLR9, LAMP-5 shuttles TLR9 from the VAMP3 ${ }^{+}$-interferon response factor signaling endosome (IRF-SE), to the LAMP-1 ${ }^{+}$pro-inflammatory-signaling endosome (PI-SE). This transition of TLR localization in turn acts as a negative regulator of IFN-1 signaling. ${ }^{28}$ Based on the known role of LAMP-5 in TLR9 localization in $\mathrm{pDC}$, we first examined the localization of intracellular LAMP-5 in MOLM-13 cells. We performed co-staining of MOLM-13 cells with antibodies against LAMP-5, LAMP-1, and myeloid differentiation primary response 88 (MYD88), a scaffold protein that is required for TLR and IL-1R signaling. Confocal microscopy showed that in MOLM-13 leukemia cells, LAMP-5 local-
A

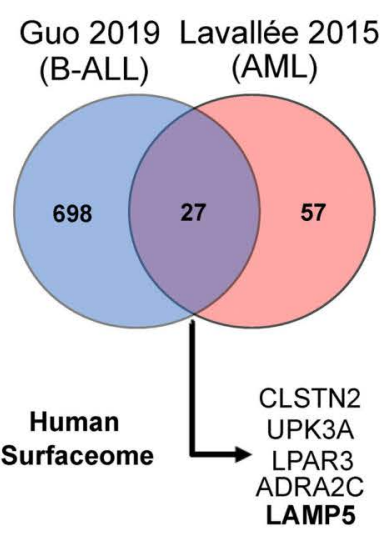

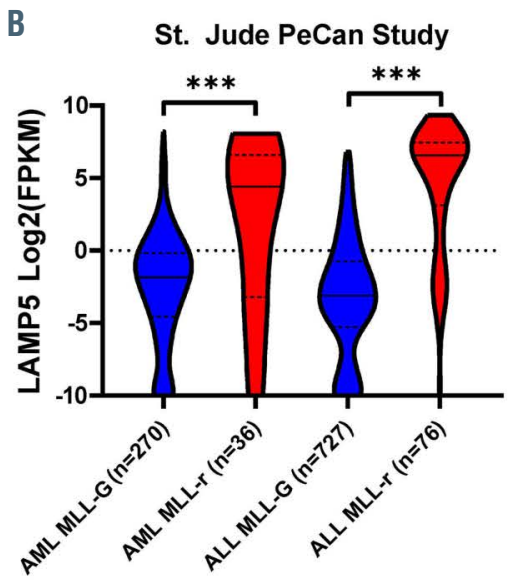

C

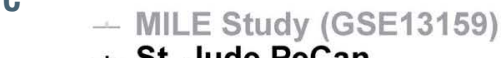
- St. Jude PeCan

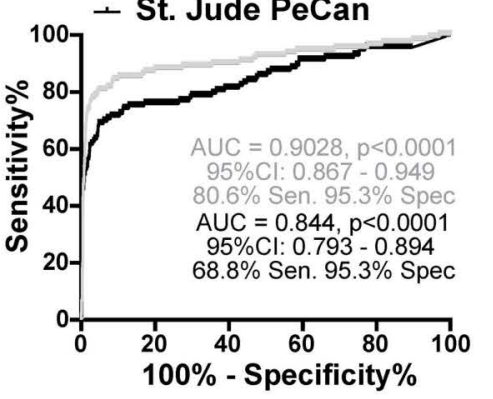

D

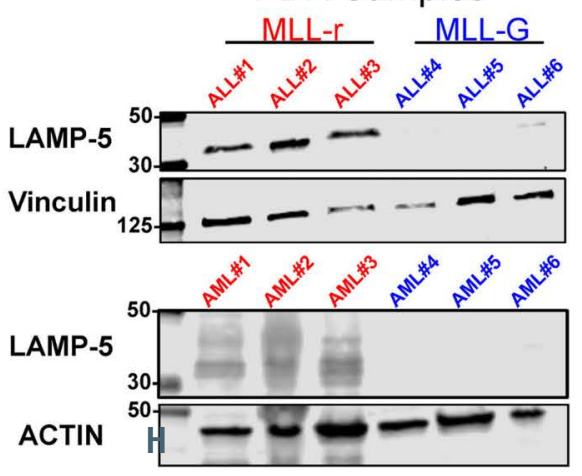

E
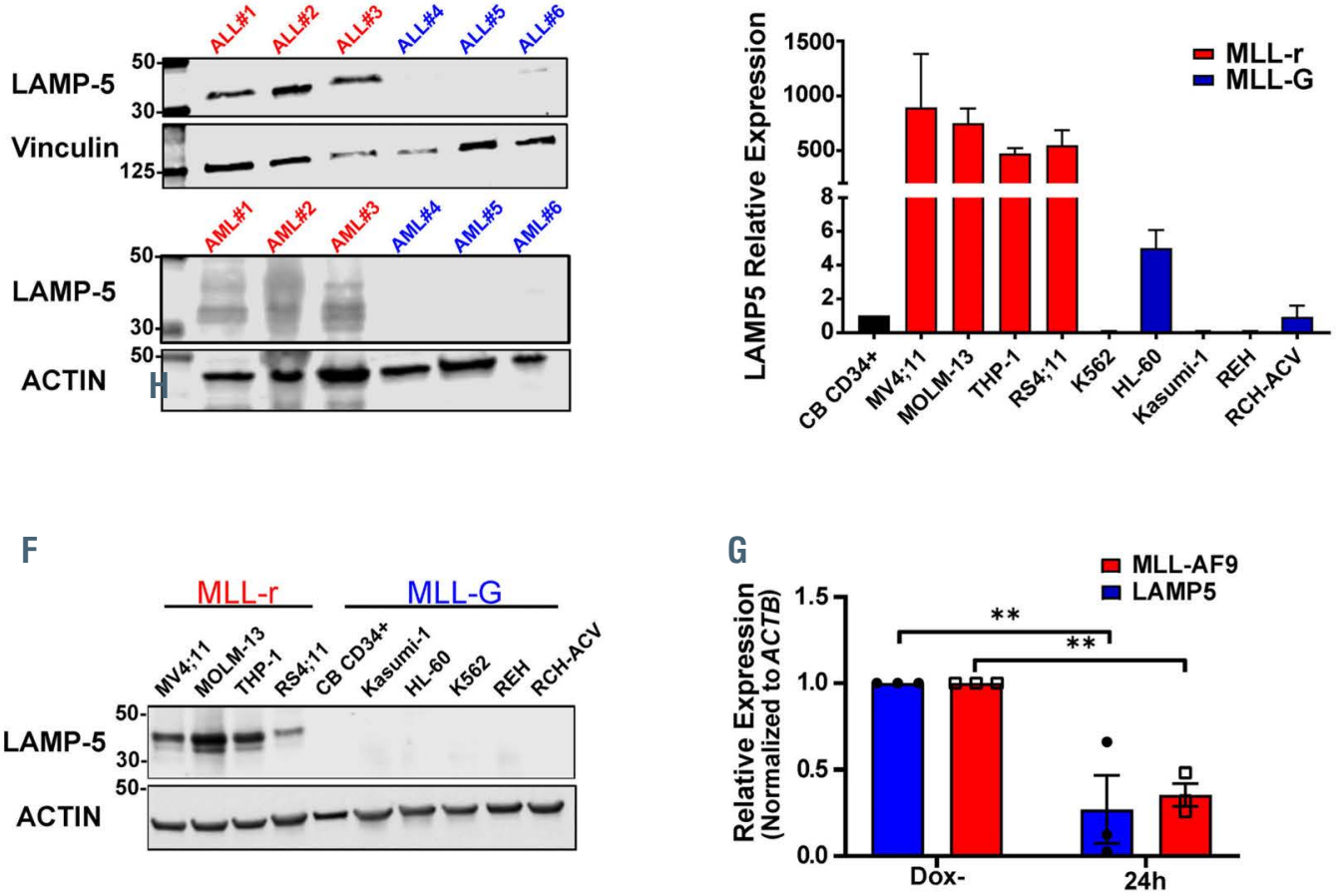

Figure 1. Continued on following page. 


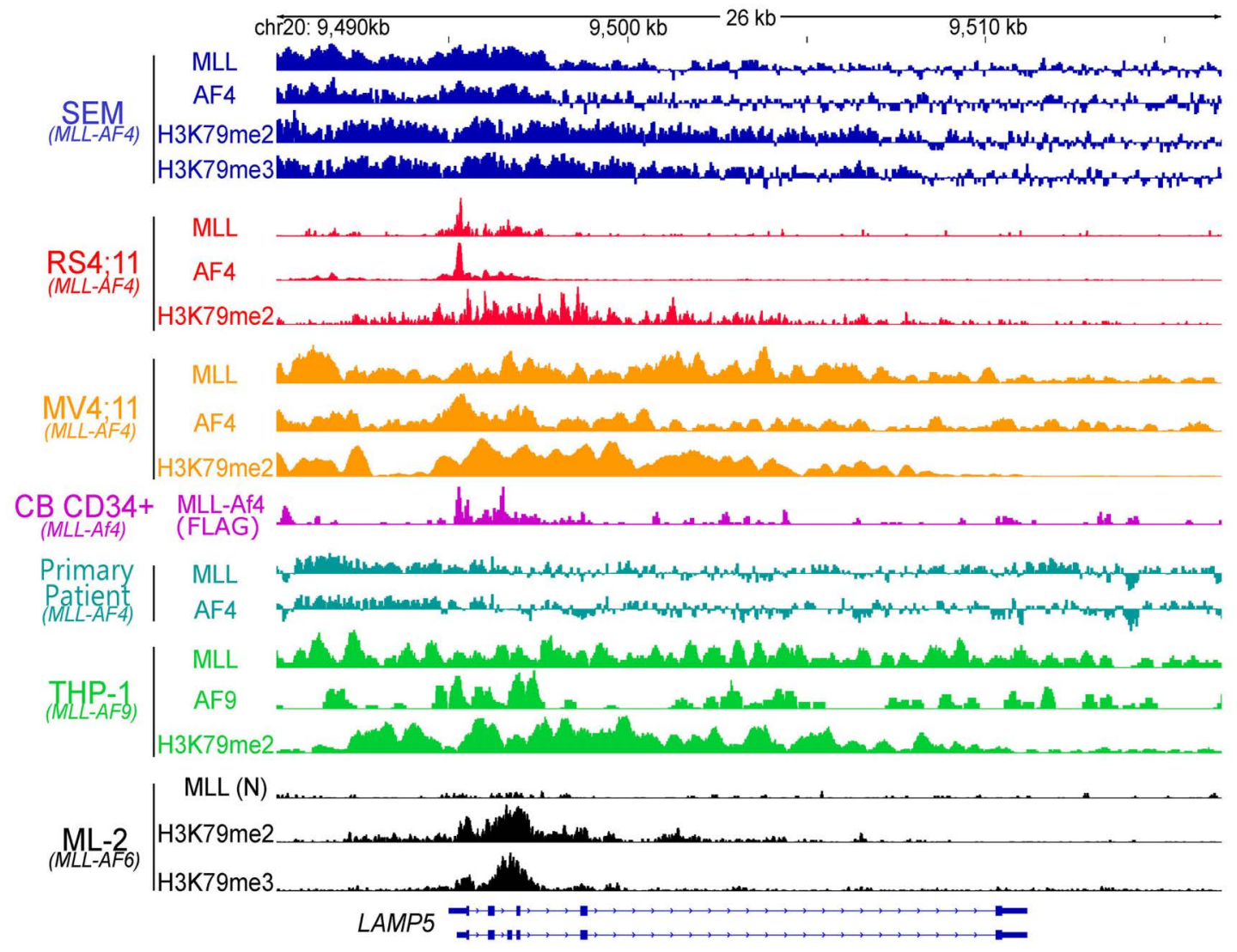

Figure 1. LAMP5 is highly expressed in mixed lineage leukemia-rearranged leukemias and is a direct target of the mixed lineage leukemia-fusion protein. (A) The intersection of published gene expression signatures composed of genes overexpressed in mixed lineage leukemia-rearranged (MLL-r) acute myeloid leukemia (AML) and acute lymphoid leukemia (ALL) when compared to MLL-germline (MLL-G) leukemias. (B) Log 2 FPKM expression of LAMP5 in AML and ALL pediatric patients with MLL-rearrangement (AML MLL-r, $n=36$ and ALL MLL-r, $n=76$ ) compared to MLL-G (AML MLL-G, $n=270$ ) (ALL MLL-G, $n=727$ ) patients. Data obtained from the St. Jude PeCan Portal and presented as median value with quartiles ( $t$-test, $* * * P<0.0001)$. (C) Receiving operating curve (ROC) analysis showing the capacity of LAMP5 to discriminate acute leukemia patients with MLL-G or MLL-r leukemias. Data obtained from GSE13159 and St. Jude Pecan Portal. (D) Western blot analysis of LAMP5 expression in pediatric primary ALL and AML samples. Actin or vinculin was used as a loading control. (E) Relative expression of LAMP5 in MLL-r leukemia (MV4;11, MOLM-13, THP-1, and RS4;11) and MLL-G leukemia (K562, HL-60, Kasumi-1, REH, RCH-ACV) cell lines. The graph represents the relative expression of LAMP5 normalized to $\beta$-ACTIN. Data are from three biological replicates. LAMP5 expression in cord blood cells was set as 1.0. Bars show mean \pm standard error of the mean (SEM). (F) Western blot analysis of the LAMP-5 levels in MLL-r leukemia and MLL-G leukemia human cell lines. CD34 ${ }^{+}$cord blood cells were used as control. Actin was used as a loading control. (G) Quantitative real-time polymerase chain reaction (RT-PCR) analysis of LAMP5 and MLL-AF9 gene expression in CD34 ${ }^{+}$cord blood cells transformed with a tetracycline-repressible MLL-AF9 construct. Gene expression was analyzed 24 hours after doxycycline incubation. Relative expression of LAMP5 and MLL-AF9 was normalized to $\beta$-ACTIN. (H) Representative chromatin immunoprecipitation sequencing (ChIP-seq) tracks at the LAMP5 locus from different MLL-r cell lines. ChIP-seq data were obtained from GSE95511 for ML-2, GSE79899 for MV4;11 and THP-1, GSE38403 for RS4;11, GSE38338 for SEM, GSE84116 for CB CD34 $4^{+}$MLL-Af4, and GSE83671 for primary patient MLL-AF4.

ized to $\mathrm{LAMP}^{-1^{+}}$vesicles. As suspected, we found MYD88 accumulating highly in the periphery of LAMP$1^{+}$vesicles in MLL-r leukemia, suggestive of TLR/IL-1R activation (Figure 3A). Conversely, in Kasumi-1 cells, MYD88 does not co-localize with LAMP-1 $^{+}$vesicles. However, overexpression of wild-type LAMP-5 in this cell line led to the relocation of MYD88 around LAMP-1 ${ }^{+}$ vesicles (Figure 3B).

We subsequently hypothesized that LAMP-5 loss may dampen TLR/IL-1R signaling in MLL-r leukemias. We thus analyzed known effector proteins downstream of TLR/IL$1 \mathrm{R}$ activation by western blot. Upon LAMP5 knockdown, we observed a reduction in phosphorylated IRAK1, NF$\kappa \mathrm{B}, \mathrm{p} 38$, and JNK, key players in the signal transduction downstream of TLR/IL-1R (Figure 3C). In order to further determine the impact of $L A M P 5$ depletion in TLR-mediat-

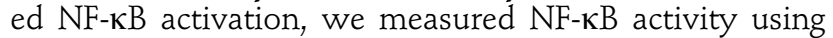
the THP-1 NF- $\kappa$ B-SEAP cell line, which contains an NF- $\kappa$ B inducible secreted embryonic alkaline phosphatase (SEAP) reporter. Robust activation of NF- $\kappa \mathrm{B}$ was evident in control cells upon incubation with PAM3CSK4 (TLR2 agonist) or LPS (TLR4 agonist). Knockdown of LAMP5 led to a near-complete blockade of this activation, suggesting that TLR-induced NF- $\mathrm{B}$ signaling is disrupted upon LAMP5 depletion (Figure 3D). Correspondingly, in Kasumi-1 cells, overexpression of LAMP5 led to increased phosphorylation of p38, JNK, and NF- $\mathrm{KB}$ along with increased cell growth (Figure $3 \mathrm{E}$ and $\mathrm{F}$ ).

A previous study showed that NF- $\kappa B$ plays a critical role in MLL-r leukemias. ${ }^{39}$ We thus hypothesized that NF$\kappa \mathrm{B}$ activation would rescue the cell growth defect seen by $L A M P 5$ depletion. We induced persistent activation of NF- $\kappa \mathrm{B}$ in leukemia cells by overexpressing a constitutively active version of inhibitor of nuclear factor $\kappa B$ kinase subunit $\beta$ (IKBKB-EE) in these cells. ${ }^{41}$ Despite sustained NF- $\kappa \mathrm{B}$ activation, knockdown of LAMP5 in MOLM-13 and RS4;11 cells still led to growth inhibition, suggesting that loss of NF- $\mathrm{KB}$ is not the only signaling event being 
affected by LAMP5 depletion (Online Supplementary Figure $S 5 A$ and $B$ ). A potential mechanism underlying this essentiality was proposed by Wang et al., where they suggested that loss of LAMPS in MLL-r leukemia led to degradation of the MLL-FP due to increased autophagy. ${ }^{42}$ However, in our experiments, we did not observe any change in the levels of the MLL-FP or LC3 A/B in THP-1 and MOLM-13 cells upon LAMP5 depletion (Online Supplemental Figure $6 A$ and $B)$. Overall, these results underscore a critical role for LAMP-5 in the activation of TLR/IL-1R signaling in MLL-r leukemia, while also indicating the presence of additional attributes that are also essential.
A

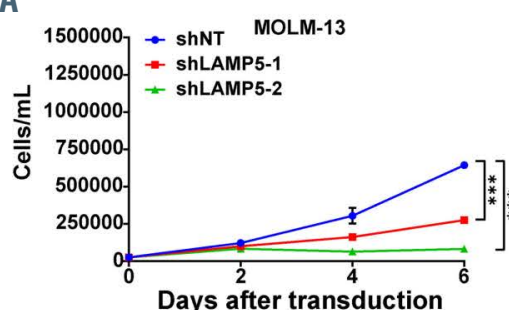

Days after transduction

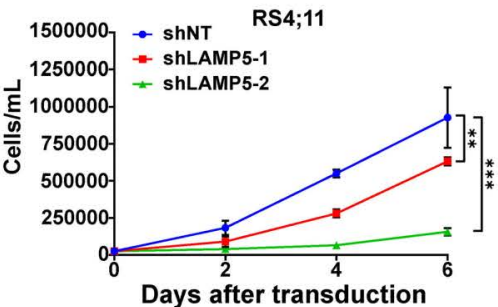

C

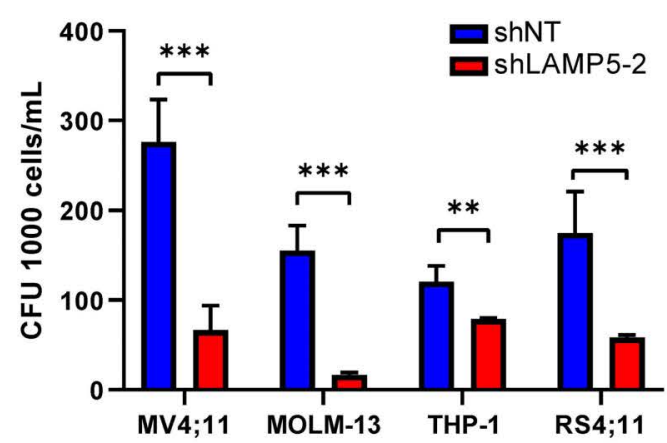

D

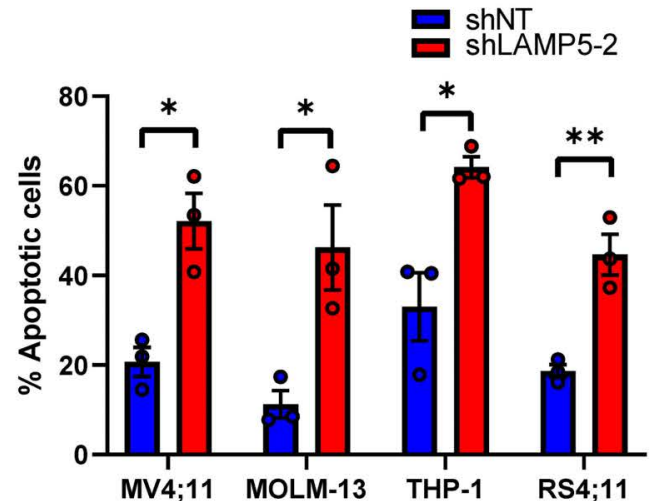

MV4;11

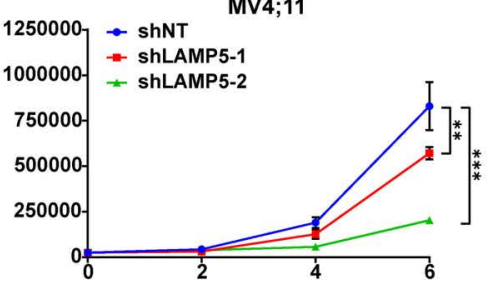

Days after transduction

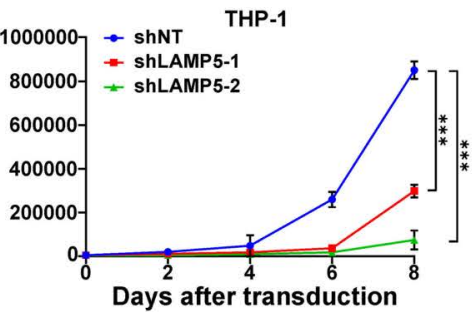

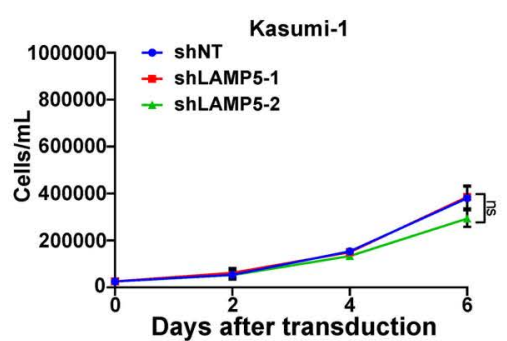

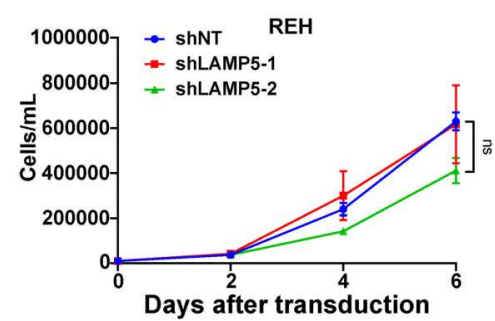

Lentivirus Venus+

ShRNA

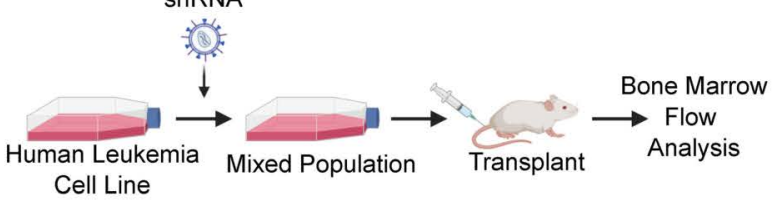

$\mathrm{F}$

MV4;11

MLL-AF10 PDX
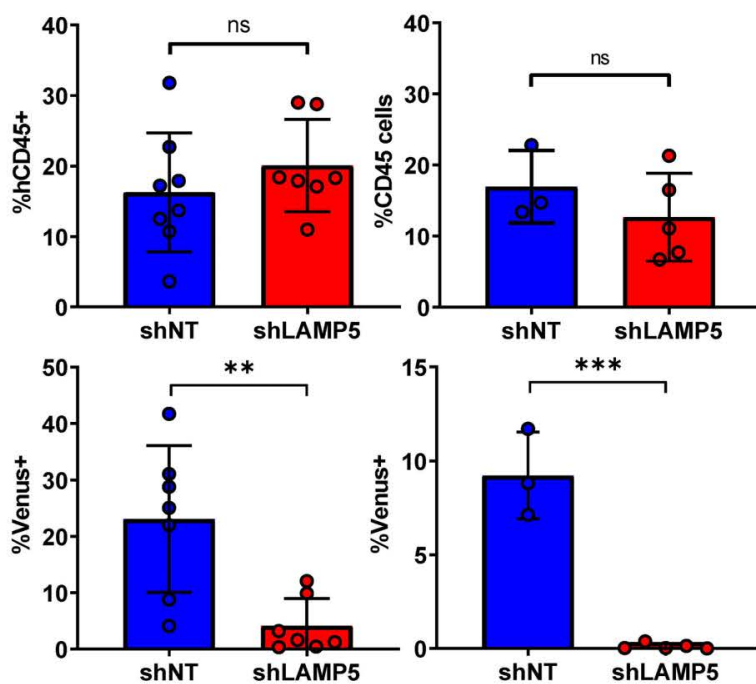

Figure 2. LAMP5 expression is required for mixed lineage leukemia-rearranged leukemia survival in vitro and in vivo. (A and B) In vitro growth of MLL-r and MLL-G leukemia cell lines (A) (MOLM-13, MV4;11, RS4;11 and THP-1) and (B) (Kasumi-1 and REH) respectively upon short hairpin RNA (shRNA) knockdown of LAMP5. Data are from three independent experiments, $t$-test, $* * P<0.01, * * * P<0.001$. (C) Colony-forming units (CFU) of MV4;11, MOLM-13, THP-1, RS4;11 cells upon LAMP5 shRNA knockdown. Data are from three biological replicates, represented as mean and SEM, $t$-test, $* * P<0.01, * * * P<0.001$. (D) Percentage of annexin $\mathrm{V}+/ 7-\mathrm{AAD}+$ cells after transduction with shNT or shLAMP5-2. Data are from three biological replicates, represented as mean and standard error of the mean (SEM) of at least three experiments. $t$-test, $* P<0.05, * * P<0.01$ (E) Schematic of in vivo xenograft transplantation. MV4;11 or MLL-AF10 PDX cells were transduced with short hairpin non-targeting control (ShNT) or shLAMP5-2 (shLAMP5) lentivirus and the mixed population of Venus ${ }^{+}$and Venus- cells were transplanted into mice. (F) Plots show the percentage of human $\mathrm{CD}^{+} 5^{+}$(upper) and Venus ${ }^{+}$cells in the CD45 fraction (lower) in MV4;11 (left) and MLL-AF10 patient-derived xenograft (PDX) sample (right) Data are from eight biological replicates for MV4;11 and five biological replicates for the MLL-AF10 PDX, represented as mean and SEM, $t$-test, $\star \star P<0.01$, $* * * P<0.001$. 
LAMP-5 is a negative regulator of interferon-1 signaling in mixed lineage leukemia-rearranged leukemias

Since activation of NF- $\mathrm{KB}$ was not sufficient to rescue the cell growth inhibition seen upon LAMP5 depletion, we next sought to understand the mechanistic significance of the inflammatory-signal-regulation function of LAMP-5 in MLL-r leukemia. In pDC, the carboxy-terminal YKHM domain of LAMP-5 was found to be required for normal localization of LAMP-5 and transportation of TLR9 from the early endosome vesicle to the pro-inflammatory vesicle. ${ }^{24,27,28}$ We thus overexpressed wild-type LAMP5 (LAMP5-WT), a Y276A mutant LAMP5 (LAMP5mut), or control vector (EV) in MV4;11 and THP-1 cells, followed by selective knockdown of endogenous LAMPS using an shRNA targeting the 3'UTR region of LAMP5 (Online Supplementary Figure S7A). Overexpression of LAMP5-WT completely prevented cell growth inhibition and apoptosis upon knockdown of endogenous LAMP5, validating LAMPS as the main target of the shRNA. In contrast, LAMP5-mut was unable to rescue cell growth or apoptosis in MV4;11 (Figure 4A and B). In pDC, LAMP5 knockdown or overexpression of LAMP5-mut induced IFN-1 activation upon TLR9-stimulation, due to retention of TLR9 in the IRF-SE. In order to determine the effect of
LAMP-5 on IFN-1 signaling in MLL-r leukemia, we turned to THP-1-ISG-SEAP cells containing an interferon-stimulated gene (ISG) inducible-SEAP reporter. Upon TLR activation by PAM3CSK4, IFN-1 signaling activation was evident only in the LAMP5-depleted cells but not in the control condition (Figure 4C). Furthermore, gene set enrichment analysis of RNA-seq from MOLM-13 cells transduced with shNT or shLAMP5-2 showed enrichment of IFN gene signatures (Online Supplementary Figure S8). Additionally, we validated the increase in IFN-1 signaling in several MLL-r cell lines by demonstrably increased expression of interferon $\alpha 2$ (IFNA2) and interferon $\beta$ (IFNB) upon depletion of LAMP5 (Figure 4D). In order to assess the role of LAMP5-depletion mediated IFN-1 activation on cell growth, we performed knockdown of interferon regulatory factor 7 (IRF7), a known regulator of IFN signaling downstream of TLR/IL1R activation, along with LAMP5 in MV4;11 and THP-1 cells. We found that loss of IRF7 alone had no significant effect on MLL-r leukemia cell growth but importantly, its depletion prevented the growth inhibition observed upon LAMP5 knockdown (Figures 4E and F; Online Supplementary Figure S9A and B). Collectively, these results demonstrate that a critical function of LAMP-5 in MLL-r leukemias is to promote the transfer of TLR/IL-1R from the IFN-1-activating signaling
A

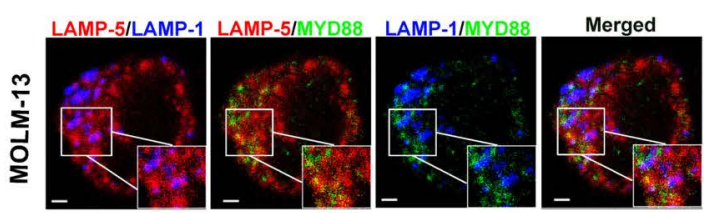

C

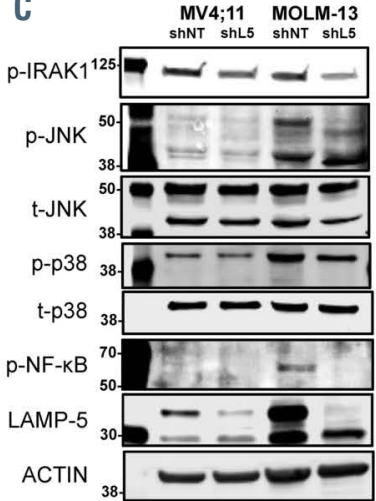

D

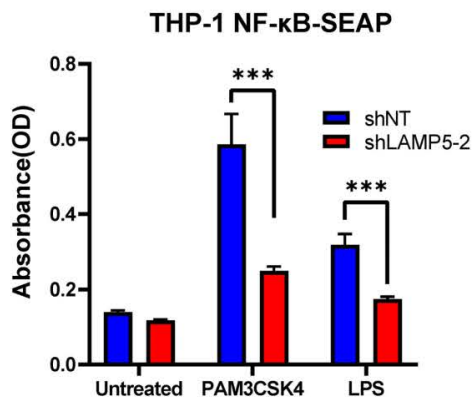

B

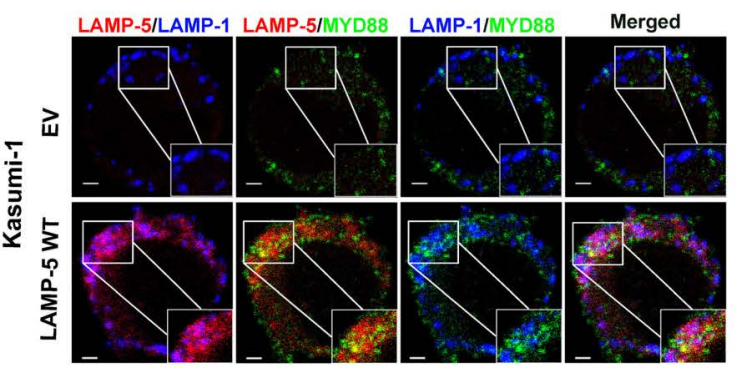

E

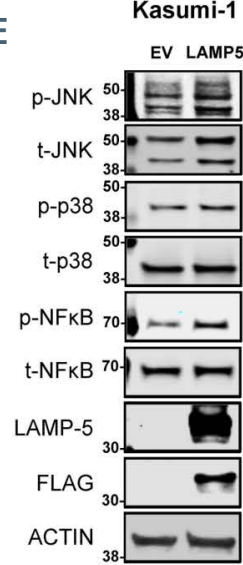

$\mathrm{F}$

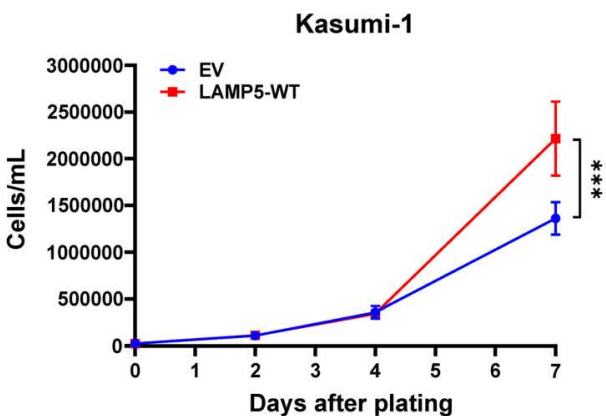

Figure 3. LAMP-5 is required for activation of Toll-like receptor/interleukin 1 receptor signaling. (A) Representative confocal microscopy images showing MOLM-13 cells stained with LAMP-5 (red), LAMP-1 (blue), and MYD88 (green); scale bar $=1 \mu \mathrm{m}$. (B) Confocal microscopy image showing Kasumi-1 cells overexpressing empty vector (EV) or wild-type LAMP5 (LAMP5-WT) stained with antibodies against LAMP-5, LAMP-1, and MYD88; scale bar $=1 \mu \mathrm{m}$. (C) Western blot analysis showing that LAMP5 depletion (shL5) led to a decrease of p-IRAK1, p-p38, p-JNK, and p-NF-KB, known downstream targets of Toll-like receptor (TLR) signaling. (D) THP-1-Blue-NF$\mathrm{KB}$ reporter cell line was treated with PAM3CSK4 $10 \mathrm{ng} / \mathrm{mL}$ or LPS $100 \mathrm{ng} / \mathrm{mL}$ in the presence or absence of LAMP-5. Data are from three independent experiments. $t$-test, $* * *, P<0.001$. (E) Western blot analysis of Kasumi-1 cells with overexpression of empty vector (EV) or LAMP5 showing increased activation of $p$-NF-KB, $p$-p38, and p-JNK. (F) In vitro cell growth of Kasumi-1 cells overexpressing EV or LAMP5-WT. Data are from three individual experiments. $t$-test, $* \star * P<0.001$. 

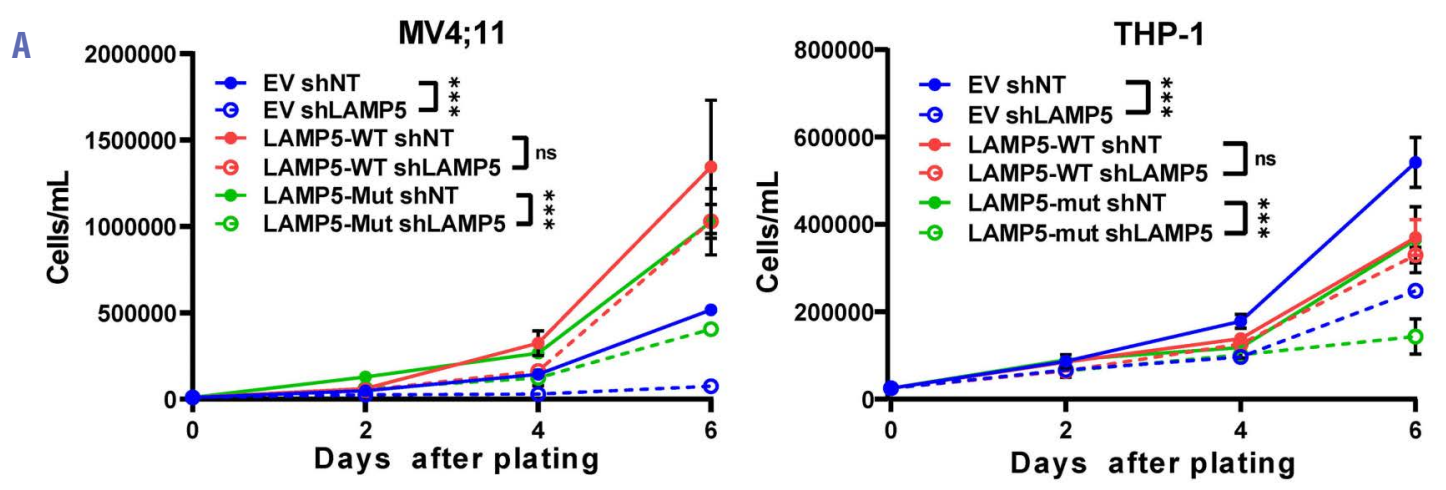

B

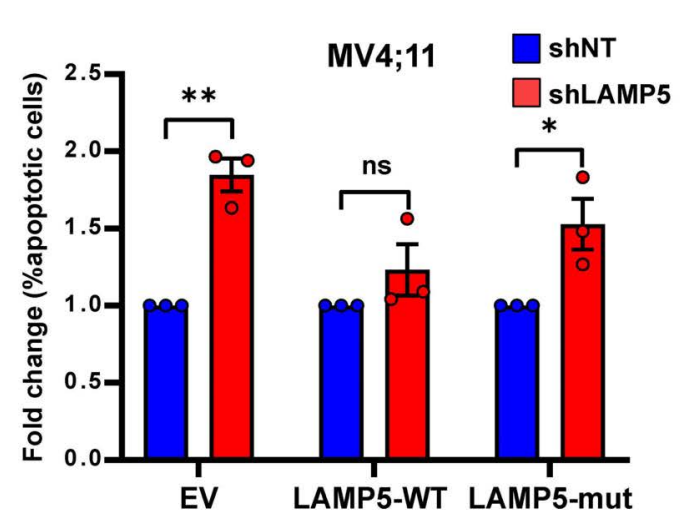

C

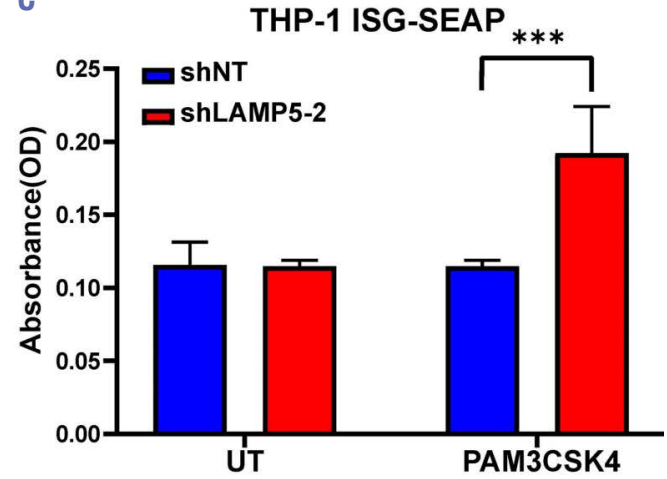

D

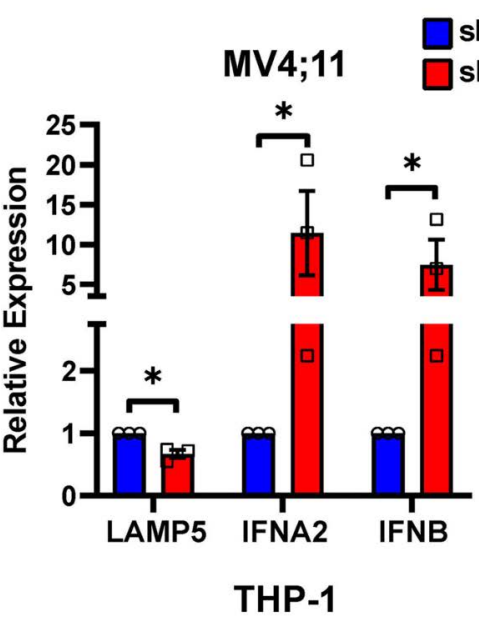

shNT

ShLAMP5-2 MOLM-13
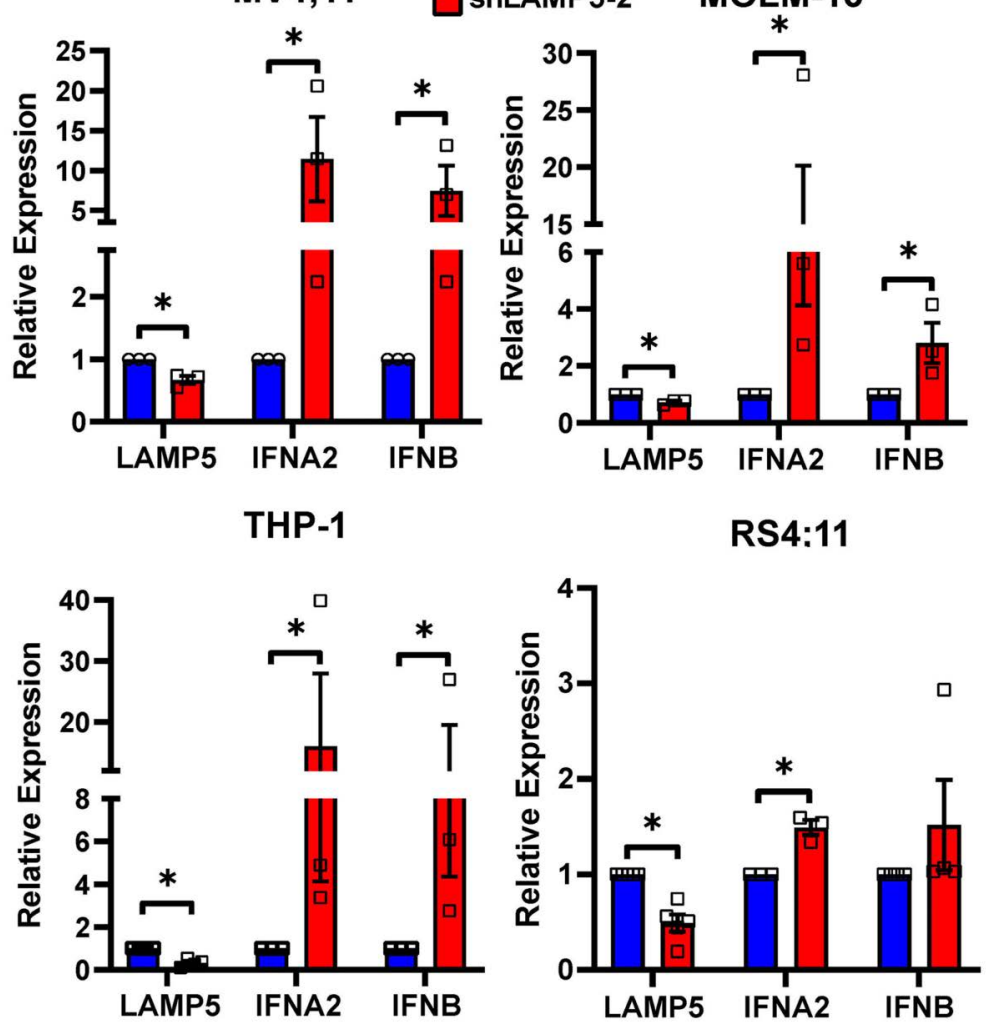

Figure 4. Continued on following page. 
E

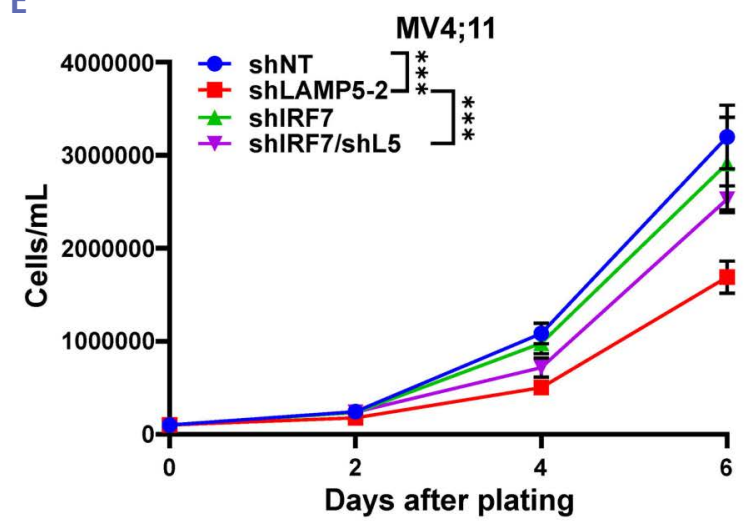

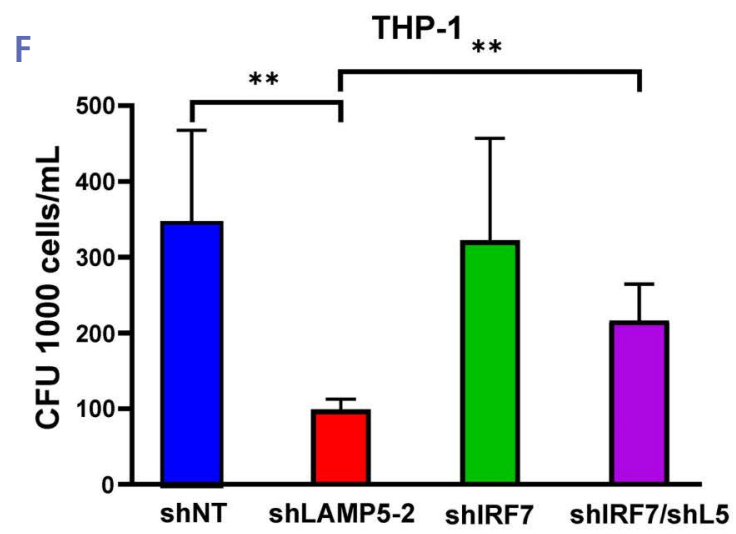

Figure 4. LAMP-5 is a negative regulator of interferon-1 signaling in mixed lineage leukemia-rearranged leukemias. (A) In vitro growth of MV4;11 and THP-1 cells overexpressing empty vector control (EV), wildtype LAMP5 (LAMP5-WT), or mutated LAMP5 (LAMP5-mut) upon shRNA knockdown of LAMP5. Data are from three independent experiments. $t$-test $* * *, P<0.001$. (B) Fold change of \% apoptotic cells in MV4;11 cell line overexpressing empty vector (EV), wild-type LAMP5 (LAMP5 WT), or mutant LAMP5 (LAMP5-mut) upon short hairpin RNA (shRNA) knockdown of LAMP5. Data are from three independent experiments. $t$-test, *P<0.05, $* \star P<0.01$. (C) THP-1 ISG blue reporter cell line was untreated (UT) or treated with $10 \mathrm{ng} / \mathrm{mL}$ PAM3CSK 4 in the presence or absence of LAMP-5. Data are from three independent experiments. Bars show mean \pm standard error of the mean (SEM). $t$-test, $* * * P<0.001$. (D) Relative expression of LAMP5, IFNA2, and IFNB upon knockdown of LAMP5 in MV4;11, MOLM-13, THP-1 and RS4;11 cells. The graph represents the relative expression of LAMP5, IFNA2, and IFNB normalized to $\beta$-actin. Data are from three biological replicates. Bars show mean \pm SEM. $t$-test *, $P<0.05$. (E) In vitro growth of MV4;11 after LAMP5, or IRF7 or LAMP5+IRF7 shRNA knockdown. Data are from three independent experiments, represented as mean and \pm standard deviation. $* * * P<0.001$. (F) Colony-forming units (CFU) of THP-1 cells upon shRNA knockdown of LAMP5, IRF7, or LAMP5+IRF7 together. Data are from three independent experiments, represented as mean and \pm SEM. $t$-test $* *, P<0.01$.

cascade to the pro-inflammatory signaling cascade. Depletion of $L A M P 5$ thus leads not only to loss of NF- $\mathrm{BB}$ activation but also to activation of IFN-1-signaling, the latter inducing cell death.

\section{Surface LAMP-5 can be detected and targeted with antibody drug conjugate therapy}

LAMP-5 has been found to briefly localize in the plasma membrane of cortical neurons in mice and is highly predicted to reach the cell membrane based on the human surfaceome. ${ }^{24,29}$ We thus sought to confirm if LAMP-5 was expressed on the surface of MLL-r leukemia cells. Using an antibody targeting the $\mathrm{N}$-terminus of LAMP-5, we were able to detect LAMP-5 on the surface of MLL-r leukemia cell lines, while none was detected in the MLL-G leukemias (Figure $5 \mathrm{~A}$ and $\mathrm{B}$ ). In order to validate the specificity of the antibody, we overexpressed $L A M P 5$ or control empty vector (EV) in Kasumi-1 cells. We detected surface LAMP-5 only in the cells that express high levels of LAMP5 (Figure 5C).

As a proof-of-concept for potential therapeutic use, we used a secondary antibody conjugated to the tubulin-toxin Mertansine, targeting the surface-LAMP-5 antibody. We observed that a 72-hour treatment with this antibody-sandwich comprised of the surface LAMP-5 antibody along with the secondary antibody drug conjugate (ADC) antibody is sufficient to reduce cell viability in MLL-r leukemia cell lines MOLM-13, RS4;11 and THP-1, while no effect was seen in Kasumi-1 cells (Figure 5D). These results suggest that LAMP-5 could be exploited as an MLL-r specific biomarker and could potentially be used as a target for immunotherapy.

\section{Discussion}

Our findings further reaffirm LAMPS as a novel and essential core gene in MLL-r leukemias, directly upregulated by the MLL-FP. Additionally, we found that one of the critical functions of LAMP-5 is to regulate innate- immune signaling in MLL-r leukemias, specifically directing the flux of activity away from IRF-SE towards the PI$\mathrm{SE}$, leading to constant activation of NF- $\kappa \mathrm{B}$ (Figure 6).

Recent discoveries have highlighted how the specific subcellular location and timing of TLR activation affect signaling outcomes in normal immune cells. ${ }^{43}$ Combes et al. showed that LAMP-5 is a negative regulator of IFN-1 signaling in $\mathrm{pDC}$ wherein it transports activated TLR9 from the IRF-SE to the PI-SE. Although dispensable for pDC cell survival, LAMP5 depletion led to unrestricted activation of IFN-1 signaling. Furthermore, aberrant expression of LAMP-5 can lead to diminished activation of $\mathrm{pDC}$ in tumors and contribute to their immunomodulatory phenotype by decreasing the IFN-1 production capacity. ${ }^{28}$ However, how these mechanisms function in leukemia is still poorly understood. Innate immune signaling and inflammation have been shown to play a crucial role in acute leukemias. ${ }^{37}$ MLL-r leukemias rely on activation of NF- $\kappa B$ downstream of TLR/IL-1R to maintain the MLL-FP gene signature and block cell differentiation. ${ }^{39,40}$ Furthermore, it has been shown that treatment with IFN-1 or activation of IFN-1 signaling is deleterious for MLL-r leukemias. ${ }^{44}$ In our study, we describe a novel role for LAMP-5 in maintaining NF- $\mathrm{KB}$ activation and blocking IFN-1 signaling downstream of TLR/IL-1R in MLL-r leukemias. We show that LAMP-5 acts as a molecular switch to maintain active TLR/IL-1R signaling in the pro-inflammatory endosome leading to NF- $\mathrm{BB}$ activation, whereas $L A M P 5$ depletion leads to activation of IFN-1 signaling and cell death. This suggests that both the LAMP-5-mediated induction of pro-inflammatory signaling and inhibition of IFN-1 signaling contribute to the pathogenesis of MLL-r leukemias. We confirmed that activation of IFN-1 signaling upon LAMP-5 depletion was deleterious for leukemia propagation, and that by depleting IRF7, cell growth and clonogenicity were rescued in $L A M P 5$-depleted cells. This suggests that increased IFN-1 signaling is at least partly responsible for inducing cell death upon $L A M P 5$ depletion. Additionally, overexpression of LAMP5 in MLL-G leukemia led to increased acti- 
vation of NF- $\mathrm{B}, \mathrm{p} 38$, and JNK, and increased cell growth, which suggests that this signaling pathway might be contributing to the therapy-resistant phenotype of MLL-r leukemias.

In humans, $L A M P 5$ expression is generally restricted to the brain and blood. In blood, LAMP5 is exclusively expressed in nonactivated $\mathrm{pDC},{ }^{27}$ wherein LAMP-5 resides in the ERGIC compartment and is transported to endo-lysosomal vesicles upon TLR9 activation. ${ }^{27,28} \mathrm{We}$ found that the aberrant increased expression of LAMP-5 in MLL-r leukemia leads to its accumulation in the plasma membrane, as demonstrated by a novel LAMP-5 antibody targeting the $\mathrm{N}$-terminus of the protein. The detec- tion of LAMP-5 on the surface of MLL leukemias provides the opportunity to potentially use it as a target for immunotherapy in this treatment-refractory malignancy. Furthermore, LAMP-5 is highly expressed in other cancers such as multiple myeloma (MM) and blastic plasmacytoid dendritic cell neoplasm (BPDCN). ${ }^{45,46}$ Therefore, LAMP-5 immunotherapies could benefit other blood diseases. Finally, total loss of Lamp5 had no major effects on the health or lifespan of mice, only causing minor behavioral effects like deficits in olfactory discrimination and increased startle response to auditory and tactile stim$\mathrm{uli}^{25,26}$ suggesting that there could be a wide therapeutic window for LAMP-5-directed therapies in humans.

A
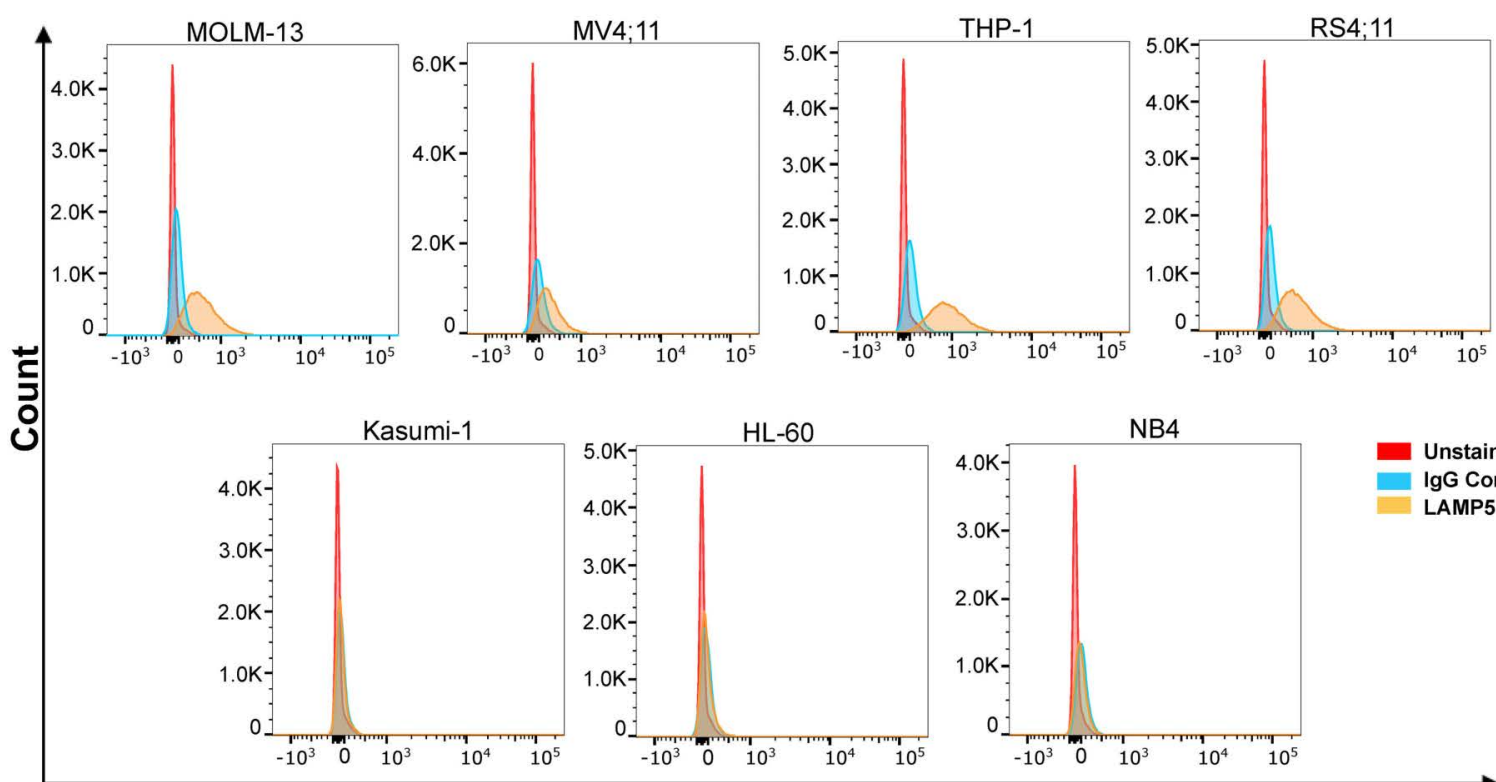

Surface LAMP-5

B

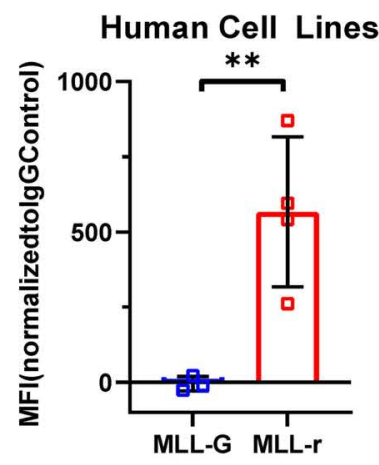

C
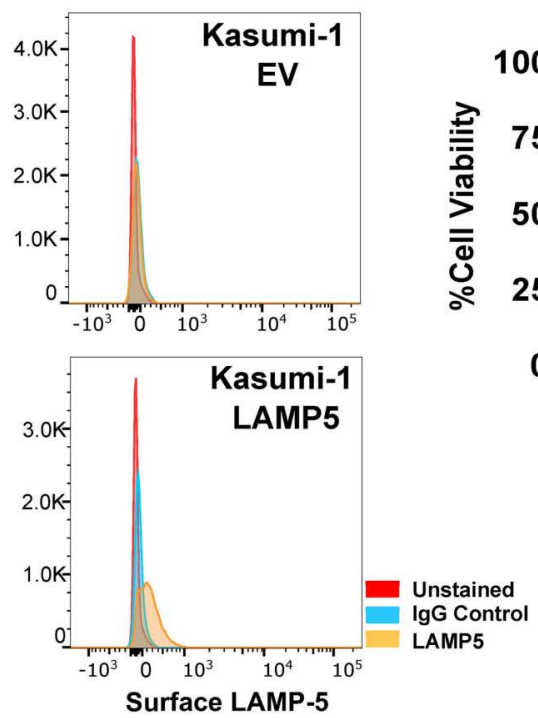

D

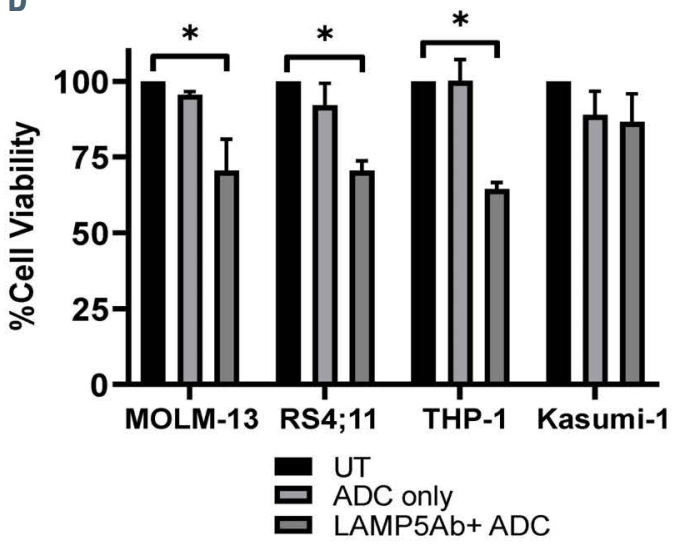

Figure 5. Surface LAMP-5 can be detected and targeted with antibody drug conjugate therapy. (A) Representative histogram plots showing LAMP-5 surface expression in mixed lineage leukemia-rearranged (MLL-r) leukemia (MOLM-13, RS4;11, MV4;11, and THP-1) and MLL-germline (MLL-G) leukemia (Kasumi-1, HL-60, and NB4) cell lines. (B) Graph showing mean fluorescence intensity (MFI) of LAMP-5 surface staining in MLL-r leukemias vs. MLL-G leukemias represented as mean and \pm standard deviation (SD). $t$-test, $* * P<0.01$. (C) Representative histogram of LAMP-5 staining in Kasumi-1 expressing empty vector (EV) or LAMP5, confirming the specificity of the antibody. (D) MOLM-13, RS4;11, THP-1, and Kasumi-1 cells were incubated with surface LAMP-5 antibody clone D1 and $\alpha$ MFc-NC-DM1 antibody drug conjugate (ADC) antibody for 72 hours. Bar graph represents cell viability from three biological replicates presented as mean and $\pm \mathrm{SEM}$. $t$-test, $* P<0.05$. 


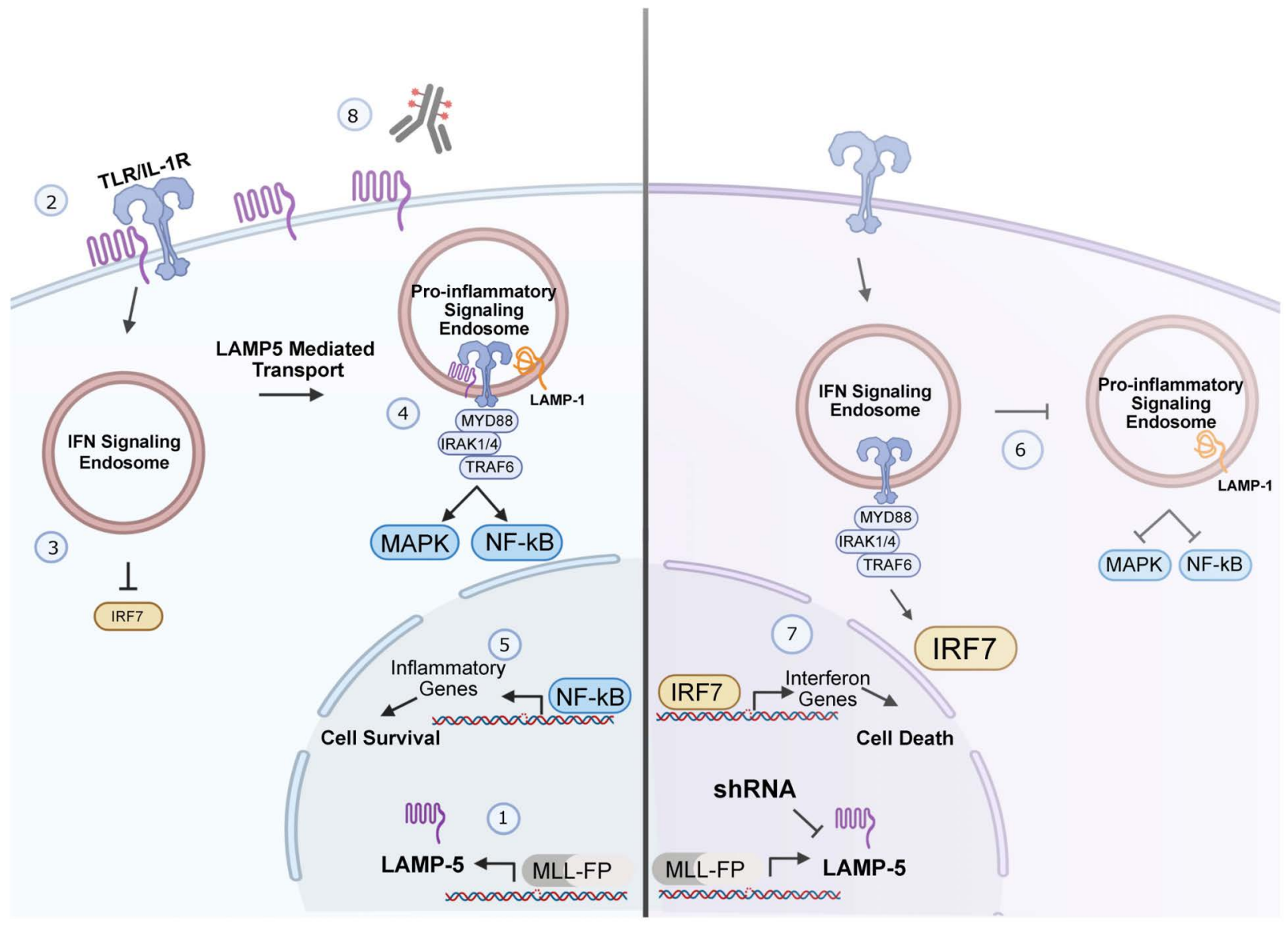

Figure 6. Proposed model to illustrate the mechanism of action of LAMP-5 in mixed lineage leukemia-rearranged leukemias and potential immunotherapy usage Left panel: 1. The mixed lineage leukemia (MLL)-FP induces expression of LAMP-5. 2. LAMP-5 gets internalized from the cell surface to the interferon signaling endosome (IFN-SE), 3. and 4. LAMP-5 is quickly shuttled to the LAMP-1 ${ }^{+}$pro-inflammatory signaling endosome (PI-SE), activating NF- $\mathrm{KB}$ signaling. 5. NF-KB activates proinflammatory signaling. Right panel: 6 . depletion of LAMP-5 leads to blockage of transport of TLR to PI-SE, with retention in and activation of the IFN-SE and 7. induction of interferon related genes and cell death. 8. Surface-LAMP-5 can be targeted in mixed lineage leukemia-rearranged (MLL-r) leukemias with immunotherapies.

Similar to our observations, Wang et al. recently showed that LAMP-5 is essential for the survival of MLLr leukemias in vitro and in vivo using shRNA knockdown. However, they propose that LAMP-5 is a negative regulator of autophagy leading to MLL-FP stabilization. They show that LAMP-5 and ATG5 co-localize in MLL-r leukemia cells and that blockade of autophagy is sufficient to rescue the increased levels of apoptosis after $L A M P 5$ knockdown. ${ }^{42}$ We were unable to detect any significant change in the levels of the MLL-FP or LC3A/B upon $L A M P 5$ knockdown. Since TLR-mediated innate immune signaling can regulate autophagy, the function of LAMP- 5 in regulating autophagy as described by Wang et al. may be downstream of its impact on endosome-lysosome trafficking. ${ }^{47}$ On the other hand, it is also possible that these effects are not directly linked, and that LAMP5 might exert its growth-promoting effects in MLL-r leukemia by multiple mechanisms. It is notable; however, that the role of autophagy in leukemia is controversial. In murine MLL leukemia models, heterozygous loss of Atg5 leads to increased leukemia cell proliferation in vitro and more aggressive leukemia in vivo, while homozygous loss is lethal to these cells. ${ }^{48}$ Additionally, while some studies suggest that Atg5-dependent autophagy may contribute to the development of MLL-AF9 driven leukemia but dispensable for propagation and chemosensitivity, others suggest that Atg5-dependent autophagy is dispensable altogether. ${ }^{49,50}$ Overall, our results show that LAMP-5 localizes both on the surface and in LAMP-1 ${ }^{+}$endosomes in leukemia, leading to constitutive activation of proinflammatory signaling, and dampening of interferon-signaling and that it can be used as a target for immunotherapy.

\section{Disclosures}

No conflicts of interest to disclose.

\section{Contributions}

GGM, LHL and ARK contributed to study conception and design; GGM, JC, $M B$ and BG acquired data; GGM, JC, $M W, D L, P P, E G$ and LHL analyzed and interpreted data; NS and LHL analyzed and interpreted RNA-seq data; GGM, JC, LHL and ARK wrote and revised the manuscript; GGM, JC, $D L, P P E$ and ARK reviewed the manuscript; MW, DL and JC provided administrative, technical, or material support.

\section{Acknowledgments}

We would like to thank Daniel Starczcynowski, Ph.D., for his intellectual input. We thank J. Bailey and V. Summey for assistance with transplantations (Comprehensive Mouse and Cancer Core at CCHMC). We would like to acknowledge the assistance of the Research Flow Cytometry Core in the Division of Rheumatology at Cincinnati Children's Hospital Medical Center. All flow cytometric data were acquired using equipment 
maintained by the Research Flow Cytometry Core in the Division of Rheumatology at Cincinnati Children's Hospital Medical Center.

\section{Funding}

The SH800S is supported by an NIH Shared Instrumentation Grant (S10OD023410). MW was supported by an NIH grant (R50 CA211404). NS was supported by an
NIH grant (R01 CA226802). PP and EV were supported by the Institut National du Cancer (INCA) (PLBIO17-187), Canceropole Paca GEFLUC (RAK18024AAA), and Fondation ARC PJA (20131200330). GGM was supported by the Chateaubriand Fellowship. ARK was supported by a Hyundai Hope on Wheels grant. LHL is a St. Baldrick's Foundation Scholar and is supported by grants from CancerFree KIDS and the NIH (L40 HL143713-01).

\section{References}

1. Meyer C, Burmeister T, Gröger D, et al. The MLL recombinome of acute leukemias in 2017. Leukemia. 2018;32(2):273-284.

2. Hilden JM, Dinndorf PA, Meerbaum SO, et al. Analysis of prognostic factors of acute lymphoblastic leukemia in infants: report on CCG 1953 from the Children's Oncology Group. Blood. 2006;108(2):441451.

3. Pieters R, Schrappe M, de Lorenzo P, et al. A treatment protocol for infants younger than 1 year with acute lymphoblastic leukaemia (Interfant-99): an observational study and a multicentre randomised trial. Lancet. 2007;370(9583):240-250.

4. Bauer J, Nelde A, Bilich T, Walz JS. Antigen targets for the development of immunotherapies in leukemia. Int $\mathrm{J} \mathrm{Mol}$ Sci. 2019;20(6):1397.

5. Jacoby E, Nguyen SM, Fountaine TJ, et al. CD19 CAR immune pressure induces Bprecursor acute lymphoblastic leukaemia lineage switch exposing inherent leukaemic plasticity. Nat Commun. 2016;7(1):12320.

6. Rayes A, McMasters RL, O'Brien MM. Lineage switch in MLL-rearranged infant leukemia following CD19-directed therapy. Pediatr Blood Cancer. 2016;63(6):11131115.

7. Haddox CL, Mangaonkar AA, Chen D, et al. Blinatumomab-induced lineage switch of B-ALL with $\mathrm{t}(4: 11)$ (q21;q23) KMT2A/AFF1 into an aggressive AML: pre- and post-switch phenotypic, cytogenetic and molecular analysis. Blood Cancer J. 2017;7(9):e607.

8. Balducci E, Nivaggioni V, Boudjarane J, et al. Lineage switch from B acute lymphoblastic leukemia to acute monocytic leukemia with persistent $t(4 ; 11)$ (q21;q23) and cytogenetic evolution under CD19-targeted therapy. Ann Hematol. 2017:96(9):1579-1581.

9. Wölfl M, Rasche M, Eyrich M, Schmid R, Reinhardt D, Schlegel PG. Spontaneous reversion of a lineage switch following an initial blinatumomab-induced ALL-toAML switch in MLL-rearranged infant ALL. Blood Adv. 2018;2(12):1382-1385.

10. Aldoss I, Song JY. Extramedullary relapse of KMT2A(MLL)-rearranged acute lymphoblastic leukemia with lineage switch following blinatumomab. Blood. 2018; 131(22):2507.

11. He RR, Nayer Z, Hogan $M$, et al. Immunotherapy- (Blinatumomab-) related lineage switch of KMT2A/AFF1 rearranged B-lymphoblastic leukemia into acute myeloid leukemia/myeloid sarcoma and subsequently into B/myeloid mixed phenotype acute leukemia. Case Rep Hematol. 2019;2019:7394619

12. Fournier E, Inchiappa L, Delattre C, et al. Increased risk of adverse acute myeloid leukemia after anti-CD19-targeted immunotherapies in KMT2A -rearranged acute lymphoblastic leukemia: a case report and review of the literature. Leuk Lymphoma. 2019;60(7):1827-1830.

13. Godfrey L, Crump NT, O'Byrne S, et al. H3K79me2/3 controls enhancer-promoter interactions and activation of the pan-cancer stem cell marker PROM1/CD133 in MLL-AF4 leukemia cells. Leukemia. 2020;35(1):90-106.

14. Li D, Hu Y, Jin Z, et al. TanCAR T cells targeting CD19 and CD133 efficiently eliminate MLL leukemic cells. Leukemia. 2018;32(9):2012-2016.

15. Lopez-Millan B, Sanchéz-Martínez D Roca-Ho H, et al. NG2 antigen is a therapeutic target for MLL-rearranged B-cell acute lymphoblastic leukemia. Leukemia. 2019;33(7):1557-1569.

16. Ma X, Liu Y, Liu Y, et al. Pan-cancer genome and transcriptome analyses of 1,699 paediatric leukaemias and solid tumours. Nature. 2018;555(7696):371-376.

17. Haferlach T, Kohlmann A, Wieczorek L, et al. Clinical utility of microarray-based gene expression profiling in the diagnosis and subclassification of leukemia: report from the International Microarray Innovations in Leukemia Study Group. J Clin Oncol. 2010;28(15):2529-2537.

18. Zangrando A, Dell'orto MC, te Kronnie G, Basso G. MLL rearrangements in pediatric acute lymphoblastic and myeloblastic leukemias: MLL specific and lineage specific signatures. BMC Med Genomics. 2009;2(1):36

19. Roychoudhury J, Clark JP, GraciaMaldonado G, et al. MEIS1 regulates an HLF-oxidative stress axis in MLL-fusion gene leukemia. Blood. 2015;125(16):25442552 .

20. Faber J, Krivtsov AV, Stubbs MC, et al. HOXA9 is required for survival in human MLL-rearranged acute leukemias. Blood. 2009;113(11):2375-2385

21. Valk PJ, Verhaak RG, Beijen MA, et al. Prognostically useful gene-expression profiles in acute myeloid leukemia. $N$ Engl Med. 2004;350(16):1617-1628.

22. Ross ME, Mahfouz R, Onciu M, et al. Gene expression profiling of pediatric acute myelogenous leukemia. Blood. 2004; 104(12):3679-3687

23. Stam RW, Schneider P, Hagelstein JAP, et al. Gene expression profiling-based dissection of MLL translocated and MLL germline acute lymphoblastic leukemia in infants. Blood. 2010;115(14):2835-2844.

24. David A, Tiveron M-C, Defays A, et al. BAD-LAMP defines a subset of early endocytic organelles in subpopulations of cortical projection neurons. J Cell Sci. 2007; 120(2):353-365.

25. Tiveron M-C, Beurrier C, Céni C, et al. LAMP5 fine-tunes GABAergic synaptic transmission in defined circuits of the mouse brain. PLoS One. 2016;11(6): e0157052.
26. Koebis M Urata S, Shinoda $Y$, et al. LAMP5 in presynaptic inhibitory terminals in the hindbrain and spinal cord: a role in startle response and auditory processing. Mol Brain. 2019;12(1):20.

27. Defays A, David A, de Gassart A, et al BAD-LAMP is a novel biomarker of nonactivated human plasmacytoid dendritic cells. Blood. 2011;118(3):609-617.

28. Combes A, Camosseto V, N'Guessan P, et al. BAD-LAMP controls TLR9 trafficking and signalling in human plasmacytoid dendritic cells. Nat Commun. 2017;8(1):913.

29. Bausch-Fluck D, Goldmann U, Müller S, et al. The in silico human surfaceome. Proc Natl Acad Sci U S A. 2018;115(46):E10988E10997.

30. Lavallée V-P, Baccelli I, Krosl J, et al. The transcriptomic landscape and directed chemical interrogation of MLL-rearranged acute myeloid leukemias. Nat Genet. 2015; 47(9):1030-1037.

31. Gu Z, Churchman ML, Roberts KG, et al. PAX5-driven subtypes of B-progenitor acute lymphoblastic leukemia. Nat Genet. 2019;51(2):296-307.

32. Benito JM, Godfrey L, Kojima K, et al. MLL-rearranged acute lymphoblastic leukemias activate BCL-2 through H3K79 methylation and are sensitive to the $\mathrm{BCL}$ 2-specific antagonist ABT-199. Cell Rep. 2015;13(12):2715-2727.

33. Lin S, Luo RT, Ptasinska A, et al. Instructive role of MLL-fusion proteins revealed by a model of $\mathrm{t}(4 ; 11)$ pro-B acute lymphoblastic leukemia. Cancer Cell. 2016;30(5):737-749.

34. Prange K, Mandoli A, Kuznetsova T, et al MLL-AF9 and MLL-AF4 oncofusion proteins bind a distinct enhancer repertoire and target the RUNX1 program in 11q23 acute myeloid leukemia. Oncogene. 2017; 36(23):3346-3356.

35. Numata A, Kwok HS, Kawasaki A, et al The basic helix-loop-helix transcription factor SHARP1 is an oncogenic driver in MLL-AF6 acute myelogenous leukemia. Nat Commun. 2018;9(1):1-16.

36. Kerry J, Godfrey L, Repapi E, et al. MLLAF4 spreading identifies binding sites that are distinct from super-enhancers and that govern sensitivity to DOT1L inhibition in leukemia. Cell Rep. 2017;18(2):482-495.

37. Hemmati S, Haque T, Gritsman K. Inflammatory signaling pathways in preleukemic and leukemic stem cells. Front Oncol. 2017;7:265.

38. Cohen P. The TLR and IL-1 signalling network at a glance. J Cell Sci. 2014; 127(11):2383-2390

39. Kuo H-P, Wang Z, Lee D-F, et al. Epigenetic roles of MLL oncoproteins are dependent on NF-kB. Cancer Cell. 2013;24(4):423437.

40. Liang K, Volk AG, Haug JS, et al. Therapeutic targeting of MLL degradation pathways in MLL-rearranged leukemia. Cell. 2017;168(1-2):59-72.

41. Mercurio F, Zhu H, Murray BW, et al. IKK- 
1 and IKK-2: cytokine-activated IкB kinases essential for NF-kappaB activation. Science. 1997;278(5339):860-866.

42. Wang W-T, Han C, Sun Y-M, et al. Activation of the lysosome-associated membrane protein LAMP5 by DOT1L serves as a bodyguard for MLL fusion oncoproteins to evade degradation in leukemia. Clin Cancer Res. 2019;25(9):2795-2808.

43. Oosenbrug T, van de Graaff MJ, Ressing ME, van Kasteren SI. Chemical tools for studying TLR signaling dynamics. Cell Chem Biol. 2017;24(7):801-812.

44. Tracey L, Streck CJ, Du Z, et al. NF-кB activation mediates resistance to IFN in MLL- rearranged acute lymphoblastic leukemia Leukemia. 2010;24(4):806-812

45. Beird HC, Khan M, Wang F, et al. Features of non-activation dendritic state and immune deficiency in blastic plasmacytoid dendritic cell neoplasm (BPDCN). Blood Cancer J. 2019;9(12):99.

46. Ledergor G, Weiner A, Zada M, et al. Single cell dissection of plasma cell heterogeneity in symptomatic and asymptomatic myeloma. Nat Med. 2018;24(12):18671876.

47. Into $\mathrm{T}$, Inomata $\mathrm{M}$, Takayama $\mathrm{E}$, Takigawa T. Autophagy in regulation of Toll-like receptor signaling. Cell Signal. 2012;24(6): 1150-1162.
48. Watson A, Riffelmacher T, Stranks A, et al Autophagy limits proliferation and glycolytic metabolism in acute myeloid leukemia. Cell Death Discov. 2015;1:15008.

49. Liu Q, Chen L, Atkinson JM, Claxton DF Wang H-G. Atg5-dependent autophagy contributes to the development of acute myeloid leukemia in an MLL-AF9-driven mouse model. Cell Death Dis. 2016;7(9): e2361.

50. Chen X, Clark J, Wunderlich M, et al. Autophagy is dispensable for $\mathrm{Km} 2 \mathrm{a} / \mathrm{Mll}$ Mllt3/Af9 AML maintenance and antileukemic effect of chloroquine. Autophagy. 2017;13(5):955-966. 